\title{
Physiological Correlates of Choice-Induced Dissonance: An Exploration of HPA-Axis Responses
}

\author{
SASHA Y. KIMEL ${ }^{1 *}$, NESTOR LOPEZ-DURAN²** AND SHINOBU KITAYAMA² \\ ${ }^{1}$ Harvard University, Cambridge, MA, USA \\ ${ }^{2}$ University of Michigan, Ann Arbor, MI, USA
}

\begin{abstract}
Choice can produce a negatively arousing cognitive conflict (called dissonance), which is thought to motivate the chooser to spread their preferences for the relevant options (called Spreading of Alternatives, or SA). The current work aimed to determine the relationship between HPA-axis activity and both choice-induced dissonance and its reduction (i.e. SA) among individuals with varying cultural backgrounds. European-Americans and Asians made a choice between two equally attractive CDs either in the presence of a cue indicative of social eyes (i.e. public-choice condition) or in the absence thereof (i.e. private-choice condition). As predicted, European-Americans and Asians showed a reliable SA primarily in the private and public choice conditions, respectively. Importantly, a sharp decline of salivary cortisol was observed over the span of $30 \mathrm{~min}$, and, moreover, this decline was reliably predicted by the magnitude of SA regardless of either culture or the choice being private vs. public. These results suggest that although choice-induced dissonance is too weak to elicit an HPA-axis stress response, SA is associated with variability in the decline of salivary cortisol during the laboratory task. Copyright @ 2015 John Wiley \& Sons, Ltd.
\end{abstract}

KEY WORDS cognitive dissonance; social eyes; culture; spreading of alternatives (SA); cortisol; hypothalamic-pituitary-adrenal (HPA) axis; choice; decision making

\section{INTRODUCTION}

Psychological research over the last half century shows the potential role of dissonance arousal in evoking a choice justification effect (Festinger, 1957; Brehm, 2007; see Harmon-Jones, Amodio \& Harmon-Jones, 2009 and Kitayama, Tompson \& Chua, 2014, for recent reviews). When making a decision between two equally attractive options, individuals are assumed to experience a negatively arousing cognitive conflict (Cooper \& Fazio, 1984; Higgins, Rhodewalt \& Zanna, 1979). To reduce this dissonance, they subsequently justify their choice by spreading their original preferences such that the chosen option becomes more attractive and the rejected option becomes less so. Importantly, this "Spreading of Alternatives" (i.e. SA) is quite robust when the choice is self-threatening (Steele, 1988) and, thus, likely negatively arousing. The goal of the current investigation was to test the degree to which the hypothalamic-pituitaryadrenal axis (HPA axis), the body's major stress response system (Dickerson \& Kemeny, 2004), may be implicated in both choice-induced dissonance arousal and its reduction (i.e. SA) among individuals with varying cultural backgrounds.

\section{Culture, choice, and self-threat}

Evidence is growing that there is cross-cultural variability in the conditions in which the act of choosing threatens the self. Specifically, in European-American cultures, the self is

\footnotetext{
*Correspondence to: Sasha Y. Kimel, Harvard University, Psychology Department, 33 Kirkland Street, Cambridge, MA 02138, USA. E-mail: skimel@fas.harvard.edu

** Correspondence to: Nestor Lopez-Duran, University of Michigan, Psychology Department, 530 Church Street, Ann Arbor, MI 48109-1109, USA. E-mail: nestorl@umich.edu
}

assumed to be independent (Markus \& Kitayama, 1991), and, thus, behaviors that are performed in private, in the absence of any social constraint, are considered self-relevant and self-expressive. These behaviors become potentially self-threatening by, for example, raising questions about one's own competence or moral integrity. In support of this analysis, previous work shows that the SA effect is quite robust when the choice is made in private (Cooper \& Fazio, 1984). Importantly, however, when European-Americans are exposed to face-like pictures that appear to be watching them while making a choice, they perceive their choice to be socially constrained (Imada \& Kitayama, 2010) and, as a result, the threat to the self is likely mitigated. As may be expected, under this condition (called the "public-choice" condition because the choice is made in the presence of a cue indicating "social eyes"), the SA becomes attenuated for this group (Imada \& Kitayama, 2010; Kitayama, Snibbe, Markus \& Suzuki, 2004).

In contrast, in Asian cultures, the self is assumed to be interdependent (Markus \& Kitayama, 1991), and, thus, behaviors that are performed in public, in the presence of social eyes, are considered self-relevant and revealing of the self. These behaviors become potentially self-threatening by, for example, evoking concerns about what others may think about one's own competence or moral integrity. As may be expected, Asians show a sizable SA effect when they make choices while thinking about the preferences of those that they care about - a manipulation designed to make social eyes cognitively more salient (Kitayama et al., 2004)—or when they make a choice for someone they care about (Hoshino-Browne et al., 2005; Kimel, Grossmann \& Kitayama, 2012). Furthermore, they also show a sizable SA effect when making a choice while exposed to face-like pictures that appear to be watching them (Imada \& Kitayama, 
2010; Kitayama et al., 2004). Importantly, Asians show little or no SA when the choice is made in private, with complete anonymity, as these actions are seen as "merely personal" and, thus, of little significance to interdependent selves (Heine \& Lehman, 1997; Kitayama et al., 2004).

One potential caveat comes from a recent argument that the paradigm used - the free-choice dissonance paradigmis fraught with an artifact due to measurement error (Chen \& Risen, 2010). Specifically, in this paradigm, participants are typically asked to rank a limited set of items (e.g. ten). They are then given a choice between two items that are similarly liked (e.g. their fifth and sixth favorites) and are later asked to rank these same items again. It is assumed that the pre-choice ranking is error-free and, thus, that these two items are truly close in preference. However, these options may be similarly ranked simply due to measurement error. That is, even though the two items appear very close in ranking, their true likability might, in fact, be further away from each another than expected. In this case, the truly more favored item may indeed be selected and ranked higher in the subsequent measurement.

Depending on how reliable the measurement of preferences is, this measurement error may be minimal (Kitayama et al., 2014). That is, the more error-prone the measurement procedure is in a given study, the more likely this artifact is to compromise the validity of the SA measure. One important way to guard against this artifact is to make sure that participants take sufficient care in the ranking task. Moreover, it is important to include a control condition in which no selfthreat is expected. If the artifact should come into play, the SA should be observed in all conditions of the experiment regardless of whether self-threat is induced. Conversely, if little or no SA were observed in at least one condition, it would suggest that the measurement error in the study was negligible. Thus, the artifact due to measurement error could be ruled out as a viable explanation for non-zero SAs that might be observed in other conditions of the study. In the current work, we therefore included the specific conditions in which little or no self-threat is anticipated (i.e. the public-choice condition for European-Americans and private-choice condition for Asians) as well as allowed participants sufficient time for the ranking task.

\section{Dissonance and physiological arousal}

In the current literature, the presence of the negatively arousing state of cognitive dissonance is typically inferred from SA or other comparable measures of attitude change (Cooper \& Fazio, 1984; Kitayama et al., 2014). Thus, little is currently known about whether the self-threat assumed in this literature may be reflected in physiological stress responses including those linked to hypothalamic-pituitary-adrenal axis (HPA-axis) activity (Dickerson \& Kemeny, 2004).

Previous research examining physiological correlates of dissonance has only used measures of sympathetic nervous system (SNS) activity (e.g. heart rate, pulse-amplitude). One study examined its relationship with choice-induced dissonance and failed to obtain any evidence of SNS activation. However, this study was also unsuccessful in obtaining a reliable SA effect (Gerard, 1967), suggesting certain methodological problems (see Croyle \& Cooper, 1983). Other studies have used an induced compliance paradigm in which participants are asked to act in a way that is contrary to their own attitudes (e.g. writing a counter-attitudinal essay). These studies showed an increase in SNS activity (i.e. skin conductance) when dissonance was presumed high (Croyle \& Cooper, 1983; Elkin \& Leippe, 1986; Losch \& Cacioppo, 1990). However, there was an inconsistent relationship between SNS activity and the reduction of dissonance (Elkin \& Leippe, 1986; Elliot \& Devine, 1994). To date, physiological correlates of any kind have not been reliably examined within the free-choice dissonance paradigm. Moreover, no study — with either of the paradigms - has tested HPA-axis activity.

The HPA axis can be activated independently of the SNS, as the two systems are linked to separate networks originating in the hypothalamus and the locus ceruleous, respectively (Tsigos \& Chrousos, 2002). Although differential activation of both systems can be stimulus-specific, under conditions of stress, they function in a continuum, with the HPA axis having a higher activation threshold than the SNS (Lovallo \& Thomas, 2000; Tsigos \& Chrousos, 2002). Moreover, while SNS activity can measure arousal but not necessarily stress per se (Bjorklund, Hokfelt \& Owman, 2010), the HPA axis is responsive to more specific stressors, especially to self-threat and threats that are socially evaluative (Mason, 1968; Dickerson \& Kemeny, 2004). Since cognitive dissonance likely involves a threat to the positive evaluation of the self (Steele, Spencer \& Lynch, 1993), it may implicate the HPA axis. Moreover, this effect may be particularly pronounced when self-threat is evoked by social evaluation (e.g. for Asians in public-choice condition).

\section{Present study}

The current work explored the degree to which the HPA axis is involved in cognitive dissonance within the free-choice paradigm. Both European-American and Asian participants made a choice either in private (i.e. in the absence of "social eyes") or in public (i.e. in the presence of "social eyes"). We anticipated that European-Americans would show a reliable SA primarily in the private (vs. public) choice condition while Asians would show the effect in the public (vs. private) choice condition. We assessed salivary cortisol levels prior to, as well as 5 and $20 \mathrm{~min}$ after, the choice. Our effort to examine HPA-axis activity as a function of culture and the choice conditions was guided by two potentially competing hypotheses regarding the relative strength of the physiological effects associated with choice-induced dissonance arousal and its reduction (i.e. SA).

First, if the stress generated by choice-induced dissonance is sufficiently strong to activate the HPA axis and is not mitigated by SA, we should observe increases in salivary cortisol when self-threat is assumed to be occurring (e.g. public-choice condition for Asians and in the private-choice condition for European-Americans). Previous work examining SNS activity in dissonance-arousing conditions within other paradigms (i.e. induced compliance) showed that the 
SNS arousal accompanying dissonance is not always related to subsequent attitude change (Croyle \& Cooper, 1983; Elkin \& Leippe, 1986; Losch \& Cacioppo, 1990; Elliot \& Devine, 1994). Given this, increased cortisol levels may be expected in the critical conditions regardless of the magnitude of dissonance reduction. Alternatively, the stress reducing effect of SA may be quite potent. If this were the case, even though dissonance is aroused, the stress involved should quickly dissipate once the choice is justified. Cortisol levels may not be necessarily high in conditions in which dissonance is aroused among those who engage in SA. Thus, the magnitude of SA may mitigate HPA-axis activity in the critical conditions.

\section{METHODS}

\section{Participants}

Thirty-seven Asian/Asian-American undergraduates (16 males, 21 females, $\left.\mathbf{M}_{\mathrm{Age}}=19.25\right)$ and 40 European-American undergraduates (22 males, 18 females, $\left.\mathrm{M}_{\mathrm{Age}}=18.95\right)$ from the University of Michigan, Ann Arbor participated in exchange for a course credit or $\$ 12$. Exclusion criteria were acute or chronic infections, acute allergy, and diseases of the adrenal gland, regular use of any medication, gravidity, and actual or past mental illness. The study was approved by the Institutional Review Board at the University of Michigan, Ann Arbor.

\section{Procedure and materials}

To control for the circadian rhythm of cortisol (Kudielka, Schommer, Hellhammer \& Kirschbaum, 2004), experimental sessions were scheduled from $2 \mathrm{pm}$ to $6: 30 \mathrm{pm}$. Participants received an e-mail with guidelines to follow during the $24 \mathrm{~h}$ prior to their participation. This included not engaging in daily activities that could influence basal cortisol levels such as consuming dairy products during the $3 \mathrm{~h}$ prior to the session or eating and drinking in the $30 \mathrm{~min}$ beforehand.

Upon arrival, the study was introduced as a consumer marketing survey sponsored by a CD retailers' association that was assessing the contributions of nature versus nurture to music preferences. As shown in Figure 1, participants were first told to relax for $20 \mathrm{~min}$ in a room in which various magazines were present. At the end of this period, they rinsed out their mouth with water in order to eliminate contaminates from blood and residuals from food. They then completed a questionnaire assessing daily activities and general health conditions that might affect basal cortisol levels. Right after this (approximately $30 \mathrm{~min}$ after their arrival), the first saliva sample (time 1) was collected to measure baseline cortisol levels.

Participants then completed the standard free-choice dissonance paradigm (Brehm, 1956; Steele et al., 1993) under procedures that are expected to reduce artifacts due to measurement error (e.g. giving participants a relatively small number of options and sufficient time to rank them; Kitayama et al., 2014). Following the procedures used by Kitayama et al. (2004), participants were first randomly assigned to one of two conditions: public-choice or privatechoice. In the public-choice condition, participants were unobtrusively exposed to a poster that included schematic emotional faces whose gazes were directed towards the participant. The poster was identical to that used by Kitayama et al. (2004) and others (Na \& Kitayama, 2012; Imada \& Kitayama, 2010) and has been found to be a valid proxy for public performance (Na \& Kitayama, 2012; Imada \& Kitayama, 2010). In the private-choice condition, no poster was present.

After random assignment, participants' viewed a binder of 30 popular CDs that had been pretested to reflect college students' current preferences and asked to select 10, at their own pace, that they would like to have but did not already own. They then ranked them by preference. Next, during an alleged music preference survey, the experimenter interrupted participants to say that the company was offering them a free CD but that there were only two left in stock, from which they could pick one to keep. In each case, the two CDs were the ones that participants had rank-ordered as their fifth and sixth favorites. Five minutes after the choice, and $15 \mathrm{~min}$ after cort time 1, the participant provided a second saliva sample (cort time 2) while completing various filler tasks. Next, participants were told that the company was interested in music preferences when customers leave a store and thus, when CDs are no longer visually present. Participants then re-

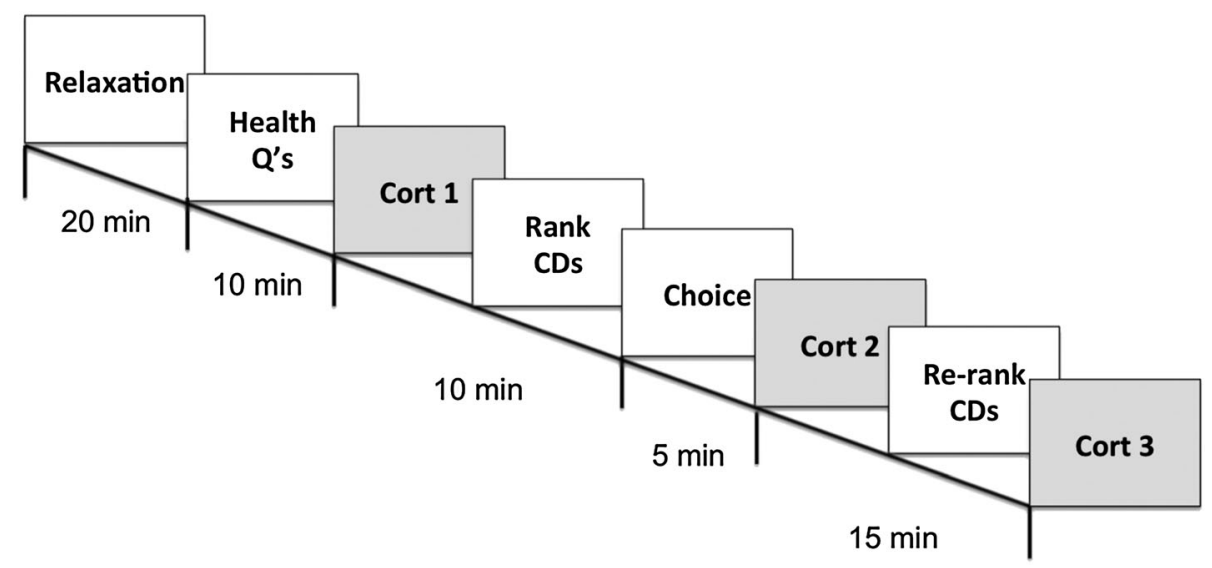

Figure 1. Timeline for the experimental procedure. Abbreviations: Cort 1 refers to Baseline Cortisol, Cort 2 was 5 min post-choice and Cort 3 was 20 min post-choice 
ranked the $10 \mathrm{CDs}$ again according to their current preferences. They were encouraged to indicate how they felt at that very moment, regardless of their previously reported preferences. At the end of the task, participants remained in the lab relaxing and provided the final saliva sample (cort time 3) $20 \mathrm{~min}$ after the choice and $30 \mathrm{~min}$ after cort time 1 . Participants then completed a demographic questionnaire and, before being debriefed, they were asked whether they noticed "anything unusual" in the room. However, no participant reported any suspicion about the poster.

\section{Salivary cortisol procedures}

Participants spit into 50-ml polypropylene collection tubes (Crystalgen Inc.) at the times previously described (see Figure 1). To stimulate saliva flow, Trident Original Flavor chewing gum was used, which has been shown to yield the least bias in steroid levels as compared to unaided saliva flow (Dabbs, 1991; see also Shirtcliff, Granger, Schwartz \& Curran, 2001). Immediately following each subject's session, saliva samples were stored in a freezer at approximately $-20{ }^{\circ}$ C. Samples were freed from mucopolysaccarides and other residuals by three freeze-thaw cycles with subsequent centrifugation, and later assayed for cortisol. Salivary cortisol levels were determined by solid-phase ${ }^{125}$ I radioimmunoassays (Coat-A-Count, Diagnostic Products Corp. Biermann, Bad Nauheim, Germany). Cortisol was measured using 400- $\mu 1$ saliva samples in combination with water-diluted standards (analytical range: 0.5 to $50 \mathrm{ng} / \mathrm{ml}$ ) and overnight incubation at room temperature. Averaged across four assays, intra-assay coefficients of variation for these samples were $4.16 \%$. Interassay coefficients of variation were $4.04 \%$ and $2.92 \%$ for low and high pools, respectively. Moreover, inter-assays coefficients of variation were $10.84 \%$ and $4.66 \%$ for low and high lypochecks, respectively. Sensitivity of the assay (B0-3 SD) was at $0.37 \mathrm{ng} / \mathrm{ml}$. This assay protocol was developed and analytically validated by previous researchers (Schultheiss \& Stanton, 2009).

\section{Analytic strategy}

In line with previous work (Hoshino-Browne et al., 2005; Steele et al., 1993), we defined Spread of Alternatives (SA) as the increase in liking of chosen $C D$ plus the decrease in liking of rejected CD [(pre-choice rank of selected $\mathrm{CD}$ - post-choice rank of selected $\mathrm{CD})+$ (post-choice rank of rejected $\mathrm{CD}$ - pre-choice rank of rejected CD)]. Consistent with other researchers, ranking was used as the measure of liking (e.g. Kitayama et al., 2004). We first used a 2 (Asian vs. European-Americans) $\times 2$ (public-choice vs. privatechoice) ANOVA and tested the effects of culture and condition on the magnitude of SA.

We then examined the effects of SA, culture, and condition on cortisol reactivity. In this analysis, we used a multilevel growth curve modeling approach (GCM) via SAS PROC MIXED with random slopes and intercepts. We used GCM modeling instead of a Repeated Measures ANOVA because GCM does not assume independence of cortisol samples within individuals, allows us to model the correct covariate matrix, and offers significantly more statistical power when examining small samples (Gueorguieva, 2004; Matthews, Altman, Campbell \& Royston, 1990; Hruschka, Kohrt \& Worthman, 2005). Cortisol data was logtransformed due to significant skewness.

\section{RESULTS}

\section{Spread of alternatives (SA)}

A 2 (culture: European-American, Asian) $\times 2$ (condition: public, private) ANOVA preformed on mean SAs showed a significant interaction, $F(1,72)=7.62, p<.01$. The pattern is illustrated in Figure 2. As predicted, Asian participants showed a reliable SA in the public-choice condition $(M=1.52, S D=1.67), t(18)=3.96, p<.01$, but not in the private-choice condition $(M=0.11, S D=2.37), t<1$. In fact, the SA was significantly greater in the former than in the later, $F(1,35=4.18, \quad p<.05)$. In contrast, EuropeanAmericans showed a reversed pattern. As predicted, they showed a reliable $\mathrm{SA}$ in the private-choice condition $(M=1.85, S D=1.49), t(19)=5.52, p<.001$. Although the $\mathrm{SA}$ in the public-choice condition was still sizable $(M=1.10, S D=1.04), t(18)=4.59, p<.001$, it was less, albeit marginally, than the one observed in the private-choice condition, $F(1,37)=3.20, p=.08$. From a different angle, it is evident that European-Americans showed a significantly greater SA than Asians did during private choice, $F(1,36)$ $=7.45, p=.01$. The pattern was non-significantly reversed in the public-choice condition, $F<1$.

\section{Cortisol stress response}

We first tested several potential covariates on the stress response using separate models. Preliminary analysis showed that neither cortisol intercept nor diurnal slope was influenced by gender, age, current oral contraceptive use, or menstrual phase (among females). These variables were subsequently dropped. The cortisol intercept decreased as a function of the number of hours from wakening, $\beta=-0.09$,

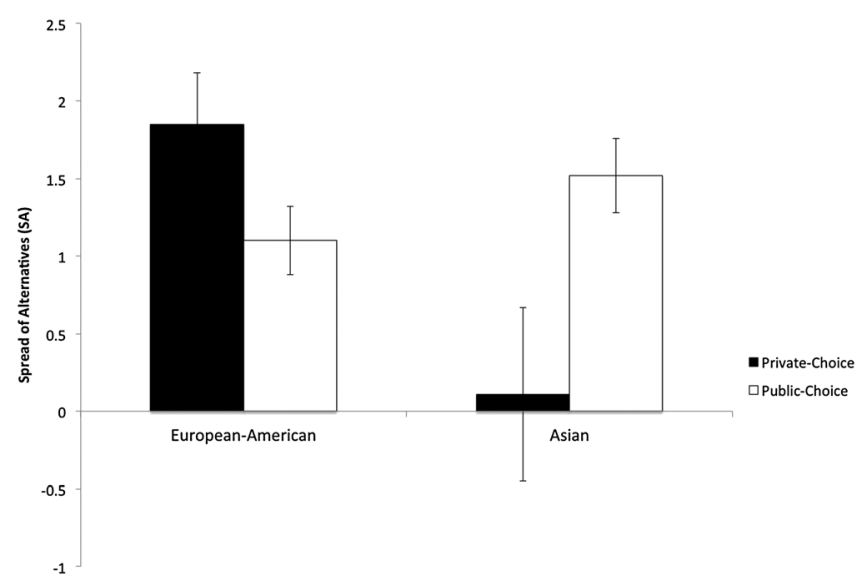

Figure 2. Spread of Alternatives (SAs) for European-American and Asian participants in the private-choice and public-choice conditions. Error bars represent standard errors of the means 
$t(62)=-3.71, p<.001$, while increasing for those who had eaten within the past $90 \mathrm{~min}, \beta=0.20, t(74)=1.97, p=.05$. These variables were entered as covariates in the subsequent analysis.

Next, we examined whether there was significant withinindividual variability in an unconditional mean model to justify the examination of predictors. There was significant variability of cortisol levels at both the intercept and the slope $(p<.001$ for both parameters). We also examined conditional linear and quadratic growth models of the cortisol trajectory from time 0 . The linear model was the best fit to the data (linear model $\mathrm{AIC}=44.5$ vs. quadratic model $\mathrm{AIC}=46.3$ ). We found a significant negative linear slope as a function of time $\beta=-0.009, t(151)=-6.66, p<.001$, indicating that cortisol levels decreased linearly over the span of 30 min during the study. Thus, we subsequently tested predictors of the linear trend only.

In testing our primary predictions, we first tested whether the cortisol slope would vary as a function of both culture and condition. Our first hypothesis implies that, if the stress generated by choice-induced dissonance is sufficiently strong to activate the HPA axis and is not mitigated by SA, we should observe increases in salivary cortisol in the public (vs. private) choice condition for Asians and in the private (vs. public) choice condition for European-Americans. However, neither of the main effects nor the interaction between the two was significant (see Table 1).

Since we observed no impact of the critical conditions (i.e. in the private-choice condition for European-Americans and the public-choice condition for Asians) we could not directly test our second hypothesis, that the effect of the critical conditions would be partially mitigated by the effect of SA. However, as shown in Figure 3, the cortisol level steadily declined over the span of $30 \mathrm{~min}$ during the experimental procedure. This cortisol decline amounted to as much as $30 \%$ from the peak response at a rate of $.10 \mu \mathrm{g} / \mathrm{dl}$ per hour.

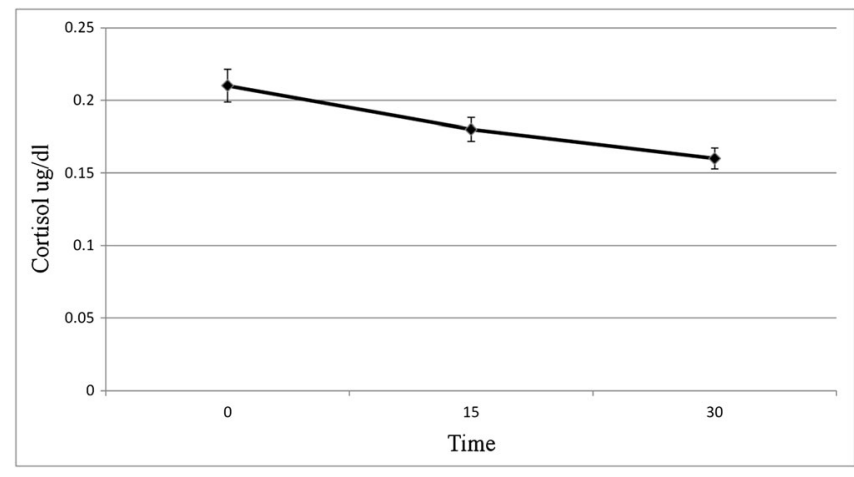

Figure 3. Salivary cortisol levels in the free-choice dissonance paradigm

This rate is nearly five-fold higher than expected from the diurnal decline (Ranjit, Young, Raghunathan \& Kaplan, 2005) and larger than might typically be expected for diurnal change during the afternoon (Edwards, Clow, Evans \& Hucklebridge, 2001). Given that it is more consistent with the rate observed during recovery to acute laboratory stressors (see Yim, Quas, Cahill \& Hayakawa, 2010; Schwabe, Haddad \& Schachinger, 2008), this decline likely reflected a recovery from events that occurred prior to arriving at the laboratory (e.g. increased stress in anticipation of the laboratory session). Therefore, we tested whether SA would entail a greater cortisol decline. This analysis showed a significant effect of SA on the slope, $F(1,124)=3.90$, $p=.05$. Specifically, increases in SA were associated with greater declines in the cortisol trajectory, $b=-.0013$, $t(124)=-1.98, p=.05$. Additional analysis that included culture and condition showed that the effect of SA on the slope was not qualified by culture, $\mathrm{SA} \times$ culture $\times$ Time $F(1,120)=.30, p>.20$, or private vs. public choice, SA $x$ Condition $\mathrm{x}$ Time $F(1,120)=.02, p>.20$.

Table 1. Individual growth curve models of cortisol $(\log )$ trajectories during a cognitive dissonance task

\begin{tabular}{lccc}
\hline Fixed effects & $\beta$ & Std. error & $t$ \\
\hline Model 1 & & & $-6.62^{* *}$ \\
Intercept & -1.16 & .176 & $2.30^{*}$ \\
Eat (no) & .216 & .093 & $-3.28^{* *}$ \\
Times since awaking & -.075 & .022 & $-4.01^{* *}$ \\
Time (slope) & -.010 & .002 & -1.07 \\
Culture (Assian) effect on baseline & -.142 & .133 & -1.54 \\
Condition (Private) effect on baseline & -.226 & .147 & 1.34 \\
Culture $\times$ condition on baseline & .261 & .194 & .55 \\
Culture effect on slope & .001 & .003 & .89 \\
Condition effect on slope & .003 & .003 & -.36 \\
Culture $\times$ condition on slope & -.001 & .004 & $-8.28^{* *}$ \\
Model 2 & & & $2.30^{*}$ \\
Intercept & -1.28 & $-3.20^{* *}$ \\
Eat (no) & -.217 & .155 & $-6.63^{* *}$ \\
Time since awaking & -.071 & .094 & -.03 \\
Time (slope) & -.007 & .022 & $-1.98^{*}$ \\
SA & -.003 & .001 & .100 \\
SA effect on slope & -.001 & .001 & \\
\hline
\end{tabular}

$\mathrm{SA}=$ spreading of alternatives 


\section{DISCUSSION}

\section{SA as a function of culture and social eyes}

The primary goal of the current work was to investigate whether HPA-axis activity would be involved in the processes of choice-induced dissonance arousal and its reduction (i.e. SA). To set the stage for addressing this question, we first identified specific conditions in which a substantial self-threat and, thus, dissonance arousal would occur. Drawing on previous work suggesting that private (vs. public) choice is self-relevant for European-Americans whereas public (vs. private) choice is more so for Asians (Kitayama et al., 2004; Na \& Kitayama, 2012), we found a significant culture by condition interaction on SA. For EuropeanAmericans, the SA was reliable in the private-choice condition (i.e. in the absence of "social eyes") whereas, for Asians, the SA was reliable in the public-choice condition (i.e. in the presence of "social eyes").

Two additional findings are relevant in interpreting the HPA-axis activity findings. First, the SA was negligible when Asians made a choice in private. This shows that, in the current study, the error associated with the measurement of preferences was negligible. Thus, the reliable SAs observed in the other conditions are unlikely to be an artifact produced by measurement error. Instead, they likely reflected substantive psychological mechanisms. The observed pattern is consistent with the hypothesis that a threat to the culturally approved sense of self (i.e. independent for EuropeanAmericans; interdependent for Asians) is instrumental in fostering SA.

Second, our manipulation of public (vs. private) choice involved no more than mere exposure to a set of schematic faces that appeared to be watching each participant. At first glance, the fact that such a subtle manipulation had substantive psychological effects might seem surprising. Nevertheless, our finding is consistent with a growing body of evidence that faces are processed both quickly and automatically to produce a psychological readiness to cope with the potentially important consequences of "being watched" (Haley \& Fessler, 2005; Na \& Kitayama, 2012; Park \& Kitayama, 2014; Rigdon, Ishii, Watabe \& Kitayama, 2009). Moreover, because of the subtlety of this manipulation, demand characteristics can be ruled out as a potential explanation of our findings.

\section{SA and cortisol stress response}

We had two potentially competing hypotheses. Our first hypothesis implies that cortisol levels should increase in conditions in which self-threat is occurring (i.e. the private-choice condition for European-Americans and the public-choice condition for Asians). However, we did not find any evidence for this hypothesis. Thus, the current data suggests that, while the self-threat operative in the free-choice paradigm is likely responsible for SA, it is not strong enough to produce a significant HPA-axis stress response. Our second hypothesis implies that SA should be associated with less HPA activation only in conditions in which the choice is self-threatening. While we observed a decline of cortisol as a function of SA, contrary to our second prediction, this was observed regardless of either culture or condition. Therefore, our findings provided no support for our second hypothesis, either.

Although our task did not result in the expected increase in cortisol typical of laboratory stress tasks, the negative correlation between SA and changes in cortisol levels during the tasks suggests that justifying one's choice is associated with variability in "recovery" to events that occurred prior to arriving to the laboratory (e.g. novelty of the laboratory visit). This conjecture is based on the observation that cortisol levels declined at a rate of $10 \mu \mathrm{g} / \mathrm{dl}$ per hour, which is in line with the kinetics observed during the recovery to acute psychosocial stressors (Yim et al., 2010; Schwabe et al., 2008). The rate of decline and the total decline of nearly $30 \%$ of maximum levels are also far greater than expected for diurnal change that occurs during the afternoon (Edwards et al., 2001; Ranjit et al., 2005). Thus, although there may be other factors that led to such high rate of decline (e.g. pulsatility of diurnal levels, post-wakening recovery among late sleepers; Young, Abelson \& Lightman, 2004), we suggest that SA likely impacted factors that modulate the down-regulation of the axis. This is consistent with research suggesting that there are individual differences in dissonance reduction processes (see Harmon-Jones, Amodio \& Harmon-Jones, 2009). It is possible that greater SA reflects better coping mechanisms or that other psychological factors (e.g. enhanced emotion regulation) facilitated the recovery to the stress of entering the laboratory (or other sources of stress leading to the decline). Our results may suggest then, that greater justification of one's choice facilitated this regulation or, conversely, that failure to justify one's choice elicited enough stress to disrupt the down regulation of the HPA axis during the study. Therefore, whether the observed effect reflects a recovery-facilitation by high SA or a recoverydisruption by low SA, or both, is yet to be determined.

\section{CONCLUSION}

The contribution of the current work was two-fold. First, we replicated previous behavioral findings on culture and choice-induced dissonance by showing that SA occurs when one's cultural self is likely threatened (i.e. when EuropeanAmericans make a private-choice and when Asians make a public-choice). Second, we found that the relationship between this phenomenon and HPA-axis activity, as assessed by salivary cortisol levels, is a complex and nuanced one. Specifically, our data suggest that the self-threat involved in choice-induced dissonance may be too subtle to produce any evidence of HPA-axis stress reactivity. At the same time, however, regardless of both culture and the choice being private or public, the magnitude of SA was significantly associated with the recovery of cortisol levels during the visit, suggesting that either those who engage in greater dissonance reduction following choice-making may have enhanced coping strategies or that failure to engage in dissonance reduction disrupted the expected declining pattern observed throughout the visit. 
More research is required to clarify the neural and physiological bases of cognitive dissonance. Existing evidence suggests that the state of dissonance is likely neutrally represented in terms of certain brain regions such as the dorsal anterior cingulate cortex (which is known to respond to cognitive conflicts) and the anterior insula (which is known to respond to negative arousal; Kitayama, Chua, Tompson \& Han, 2013). Future work should examine whether this neural representation of dissonance may be linked to the activation of neuroendocrine response systems including the HPA axis. Although we did not find any evidence for HPA-axis reactivity in the current study, such a link may exist in daily conflicts that entail much stronger dissonance reactions or in situations that involve higher levels of perceived social evaluation (e.g. Curley, Yates \& Abrams, 1986). Moreover, participants in our study were likely still regulating to the stress associated with coming to a laboratory study. Although this is a common limitation of many studies and it does not prevent HPA-axis activation to common laboratorybased paradigms (see for example Abelson et al., 2014), it is possible that elevated cortisol levels at the start of the task could limit stress reactivity to a weak stressor. Therefore, future work may benefit from a procedure that allows the HPA axis to habituate more fully in the experimental setting before dissonance is induced.

\section{REFERENCES}

Abelson, J. L., Erickson, T. M., Mayer, S. E., Crocker, J., Briggs, H., Lopez-Duran, N. L., \& Liberzon, I. (2014). Brief cognitive intervention can modulate neuroendocrine stress responses to the trier social stress test: Buffering effects of a compassionate goal orientation. Psychoneuroendocrinology, 44, 60-70. doi:10.1016/j.psyneuen.2014.02.016

Bjorklund, A., Hokfelt, T., \& Owman, C., (2010). Sympathetic nervous system. In George Fink (Ed.), Stress science: neuroendocrinology (Vol. 47, pp. 290). Oxford, UK: Academic Press.

Brehm, J. W. (2007). A brief history of dissonance theory. Social and Personality Psychology Compass, 1(1), 381-391.

Brehm, J. W. (1956). Post-decision changes in the desirability of alternatives. Journal of Abnormal and Social Psychology, 52 (2), 384-389.

Chen, M. K., \& Risen, J. L. (2010). How choice affects and reflects preferences: Revisiting the free-choice paradigm. Journal of Personality and Social Psychology, 99(4), 573-594.

Cooper, J., \& Fazio, R. (1984). A new look at dissonance theory. Advances in Experimental Social Psychology, 17, 229-264.

Croyle, R. T., \& Cooper, J. (1983). Dissonance arousal: Physiological evidence. Journal of Personality and Social Psychology, 45, 782-791.

Curley, S. P., Yates, J. F., \& Abrams, R. A. (1986). Psychological sources of ambiguity avoidance. Organizational Behavior and Human Decision Processes, 38(2), 230-256.

Dabbs, J. M. (1991). Salivary testosterone measurement: Collecting, storing and mailing salivary samples. Physiology \& Behavior, 49, 815-817.

Dickerson, S. S., \& Kemeny, M. E. (2004). Acute stressors and cortisol responses: A theoretical integration and synthesis of laboratory research. Psychological Bulletin, 130(3), 355-391.

Edwards, S., Clow, A., Evans, P., \& Hucklebridge, F. (2001). Exploration of the awakening cortisol response in relation to diurnal cortisol secretory activity. Life Sciences, 68(18), 2093-2103.
Elkin, R. A., \& Leippe, M. R. (1986). Physiological arousal, dissonance, and attitude change: Evidence for a dissonance-arousal link and a "don't remind me" effect. Journal of Personality and Social Psychology, 51, 55-65.

Elliot, A. J., \& Devine, P. G. (1994). On the motivation nature of cognitive dissonance: Dissonance as psychological discomfort. Journal of Personality and Social Psychology, 67, 382-394.

Festinger, L. (1957). A theory of cognitive dissonance. Evanston, IL: Row, Peterson.

Gerard, H. B. (1967). Choice difficulty, dissonance, and the decision sequence. Journal of Personality, 35, 91-108.

Gueorguieva, R. (2004). Move over ANOVA. Archives of General Psychiatry, 61(3), 310.

Haley, K. J., \& Fessler, D. M. T. (2005). Nobody's watching? Evolution and Human Behavior, 26(3), 245-256. doi:10.1016/j. evolhumbehav.2005.01.002

Harmon-Jones, E., Amodio, D. M., \& Harmon-Jones, C. (2009). Action-based model of dissonance: A review, integration, and expansion of conceptions of cognitive conflict. Advances in Experimental Social Psychology, 41, 119-166.

Harmon-Jones, E., \& Harmon-Jones, C. (2009). Action-based model of dissonance: A review of behavioral, anterior cingulate, and prefrontal cortical mechanisms. Social and Personality Psychology Compass, 2(3), 1518-1538.

Heine, S. J., \& Lehman, D. R. (1997). The cultural construction of self-enhancement: an examination of group-serving biases. Journal of Personality and Social Psychology, 72(6), 1268.

Higgins, E. T., Rhodewalt, F., \& Zanna, M. P. (1979). Dissonance motivation: Its nature, persistence, and reinstatement. Journal of Experimental Social Psychology, 15, 16-34.

Hoshino-Browne, E., Zanna, A. S., Spencer, S. J., Zanna, M. P., Kitayama, S., \& Lackenbauer, S. (2005). On the cultural guises of cognitive dissonance: The case of easterners and westerners. Journal of Personality and Social Psychology, 89, 294-310.

Hruschka, D. J., Kohrt, B. A., \& Worthman, C. M. (2005). Estimating between- and within individual variation in cortisol levels using multilevel models. Psychoneuroendocrinology, 30(7), 698-714

Imada, T., \& Kitayama, S. (2010). Social eyes and choice justification: Culture and dissonance revisited. Social Cognition, 28(5), 589-608.

Kimel, S. Y., Grossmann, I., \& Kitayama S. (2012). When giftgiving produces dissonance: Effects of subliminal affiliation priming on choices for one's self versus close others. Journal of Experimental Social Psychology, 48(5), 1221-1224.

Kitayama, S., Chua, H. F., Tompson, S., \& Han, S. (2013). Neural mechanisms of dissonance: An fMRI investigation of choice justification. NeuroImage, 13, 206-212.

Kitayama, S., Snibbe, A. C., Markus, H. R., \& Suzuki, T. (2004). Is there any "free" choice? self and dissonance in two cultures. Psychological Science, 15(8), 527-533.

Kitayama, S., Tompson, S., \& Chua, H. F. (2014). Cultural neuroscience of choice justification. In J. Forgas, \& E. Harmon-Jones (Eds.), Control within: Motivation and its regulation (pp. 313-330). Sydney Symposium: Psychology Press.

Kudielka, B. M., Schommer, N. C., Hellhammer D. H., \& Kirschbaum, C. (2004). Acute HPA axis responses, heart rate, and mood changes to psychosocial stress (TSST) in humans at different times of day. Psychoneuroendocrinology, 29, 983-992.

Losch, M. E., \& Cacioppo, J. T. (1990). Cognitive dissonance may enhance sympathetic tonus, but attitudes are changed to reduce negative affect rather than arousal. Journal of Experimental Social Psychology, 26, 289-304.

Lovallo, W. R., \& Thomas, T. L. (2000). Stress hormones in psychophysiological research: emotional, behavioral, and cognitive implications. In J. T. Cacioppo, L. G. Tassinary, \& G.G. Bernston (Eds.) Handbook of psychophysiology (Second ed., pp. 342-367). New York: Cambridge University Press. 
Mason, J. W. (1968). A review of psychoendocrine research on the pituitary-adrenal corticalsystem. Psychosomatic Medicine, 30, 576-607.

Markus H. R., \& Kitayama, S. (1991). Culture and the self: Implications for cognition, emotion and motivation. Psychological Review, 98, 224-253.

Matthews, J. N., Altman, D. G., Campbell, M. J., \& Royston, P. (1990). Analysis of serial measurements in medical research. British Medical Journal, 300(6719), 230-235.

Na, J., \& Kitayama, S. (2012). Will people work hard on a task they choose? Social-eyes priming in different cultural contexts. Journal of Experimental Social Psychology, 48(1), 284-290.

Park, J., \& Kitayama, S. (2014). Interdependent selves show face-induced facilitation of error processing: Cultural neuroscience of self-threat. Social Cognitive and Affective Neuroscience, 9(2), 201-208.

Ranjit, N., Young, E. A., Raghunathan, T. E., \& Kaplan, G. A. (2005). Modeling cortisol rhythms in a population-based study. Psychoneuroendocrinology, 30, 615-624.

Rigdon, M., Ishii, K., Watabe, M., \& Kitayama, S. (2009). Minimal social cues in the dictator game. Journal of Economic Psychology, 30, 358-367.

Schultheiss, O. C., \& Stanton, S. J. (2009). Assessment of salivary hormones. In E. Harmon-Jones, \& J. S. Beer (Eds.), Methods in the neurobiology of social and personality psychology (pp. 17-44). New York, NY: Guilford.

Schwabe, L., Haddad, L., \& Schachinger, H. (2008). HPA axis activation by a socially evaluated cold-pressor test. Psychoneuroendocrinology, 33, 890-895.

Shirtcliff, E. A., Granger, D. A., Schwartz, E., Curran, M. J. (2001). Use of salivary biomarkers in bio-behavioral research: Cotton-based sample collection methods can interfere with salivary immunoassay results. Psychoneuroendocrinology, 26(2), 165-173.

Steele, C. M. (1988). The psychology of self-affirmation: Sustaining the integrity of the self. Advances in Experimental Social Psychology, 21(2), 261-302.

Steele, C. M., Spencer, S. J., \& Lynch, M. (1993). Self-image resilience and dissonance: The role of affirmational resources. Journal of Personality and Social Psychology, 64(6), 885-896.

Tsigos, C., \& Chrousos, G. P., (2002). Hypothalamic-pituitaryadrenal axis, neuroendocrine factors and stress. Journal of Psychosomatic Research, 53(4), 865-871.

Yim, I. S., Quas, J. A., Cahill, L., \& Hayakawa, C. M. (2010). Children's and adults' salivary cortisol responses to an identical psychosocial laboratory stressor. Psychoneuroendocrinology, 35, 241-248.

Young, E. A., Abelson, J. L., \& Lightman, S. L. (2004). Cortisol pulsatility and its role in stress regulation and health. Frontiers in Neuroendocrinology, 25, 69-76.

Authors' biographies:

Sasha Kimel is a post-doctoral scholar at Harvard University. She earned her PhD in Social Psychology from University of Michigan. Her work focuses on understanding the psychological underpinnings of interethnic conflict.

Nestor Lopez-Duran is an Assistant Professor in Clinical Psychology at University of Michigan, where he also received his $\mathrm{PhD}$. His work focuses on understanding of the role of stress regulation in childhood mood disorders.

Shinobu Kitayama is a Professor in Social Psychology at University of Michigan, where he also received his $\mathrm{PhD}$. His current work focuses on (i) gene $\times$ culture interactions, (ii) culture and the self, and (iii) culture and biological health.

Authors' addresses:

Sasha Y. Kimel, Harvard University, Cambridge, MA, USA

Nestor Lopez-Duran and Shinabu Kitayama, University of Michigan, Ann Arbor, MI, USA 